\title{
COMITE EDITORIAL
}

Alain-Philippe Durand, University of Arizona, EUA

Christianne Benatti Rochebois, Universidade Federal de Viçosa, Brasil

Christine Preville, Université de Montréal, Canadá

Dário Pagel, Universidade Federal de Sergipe, Brasil

Denise Lino de Araújo, Universidade Federal de Campina Grande, Brasil

Elisabetta Santoro, Universidade de São Paulo, Brasil

Hebe Macedo de Carvalho, Universidade Federal do Ceará, Brasil

Helena Topa Valentim, Universidade Nova de Lisboa, Portugal

Ilza do Socorro Galvão Cutrim, Universidade Federal do Maranhão, Brasil

Isis Milreu, Universidade Federal de Campina Grande, Brasil

Joice Armani Galli, Universidade Federal de Pernambuco, Brasil

José Hélder Pinheiro Alves, Universidade Federal de Campina Grande, Brasil

José Leonardo Tonus, Universidade Paris III, França

Josilene Pinheiro-Mariz, Universidade Federal de Campina Grande, Brasil

Laura Taddei Brandini, Universidade Estadual de Londrina, Brasil

Marco Antônio M. Costa, Universidade Federal de Campina Grande

Maria Antonieta A. Herrero, UNED-Madri, Espanha

Márcia Tavares Silva, Universidade Federal de Campina Grande, Brasil

Márcio Venício Barbosa, Universidade Federal do Rio Grande do Norte, Brasil

Maria Angélica de Oliveira, Universidade Federal de Campina Grande, Brasil

Maria Auxiliadora Bezerra, Universidade Federal de Campina Grande, Brasil

Maria da Conceição Coelho Ferreira, Université Lumière Lyon II, França

Maria da Glória Magalhães dos Reis, Universidade de Brasília

Maria de Fátima Sopas Rocha, Universidade Federal do Maranhão,

Brasil Nicolas Regis Drouvot, Université de Grenoble, França

Nicole Blondeau, Université Paris VIII, França

Rita Jover Faleiros, Universidade Federal de São Paulo, Brasil

Sandra Assunção, Universidade Paris X, França

Sinara de Oliveira Branco, Universidade Federal de Campina Grande, Brasil

Wanderlan da Silva Alves, Universidade Estadual da Paraíba, Brasil

\section{COMITÊ TÉCNICO}

Aldenora M. Chaves P. Carvalho, Universidade Federal do Maranhão, Brasil Alyere Silva Farias, Universidade Federal do Rio Grande do Norte, Brasil Diana Barbosa de Freitas, Universidade Federal de Campina Grande, Brasil Francinaldo de Souza Lima, Universidade Federal de Campina Grande, Brasil Ferroudja Allouache, Université Paris VIII, França

Hernani Carvalho Veloso, Universidade Federal do Maranhão Ibéria de Souza Farias, Universidade Estadual da Paraíba, Brasil

Jéssica Pereira Gonçalves, Universidade Federal de Campina Grande Jhuliane Evelyn Silva, Universidade Federal de Campina Grande, Brasil Lino Dias Correia Neto, Universidade Federal de Campina Grande, Brasil Maria Rennally Soares da Silva, Universidade Federal de Campina Grande, Brasil Marinez Sousa Tamburini, Universidade Federal do Maranhão, Brasil

Nyeberth Emanuel Pereira dos Santos, Universidade Federal de Campina Grande, Brasil Normando Brito de Almeida, Universidade Federal de Campina Grande Patrícia Pinheiro Pinheiro Menegon, Universidade Federal do Maranhão Viviane Morais de Caldas Gomes, Universidade Federal de Campina Grande, Brasil Vívian Monteiro, Universidade Federal de Campina Grande 